\title{
Supporting Control Room Operators in Highly Automated Future Power Networks
}

Chen, Minjiang; Catterson, Victoria ; Syed, Mazheruddin; MCarthur, Stephen; Burt, Graeme M.; Marinelli, Mattia; Prostejovsky, Alexander; Heussen, Kai

\section{Published in:}

Cired - Open Access Proceedings Journal

Link to article, DOI:

10.1049/oap-cired.2017.1107

Publication date:

2017

Document Version

Peer reviewed version

Link back to DTU Orbit

Citation (APA):

Chen, M., Catterson, V., Syed, M., MCarthur, S., Burt, G. M., Marinelli, M., Prostejovsky, A., \& Heussen, K. (2017). Supporting Control Room Operators in Highly Automated Future Power Networks. Cired - Open Access Proceedings Journal, 2017(1), 1492-5. https://doi.org/10.1049/oap-cired.2017.1107

\section{General rights}

Copyright and moral rights for the publications made accessible in the public portal are retained by the authors and/or other copyright owners and it is a condition of accessing publications that users recognise and abide by the legal requirements associated with these rights.

- Users may download and print one copy of any publication from the public portal for the purpose of private study or research.

- You may not further distribute the material or use it for any profit-making activity or commercial gain

- You may freely distribute the URL identifying the publication in the public portal 


\section{SUPPORTING CONTROL ROOM OPERATORS IN HIGHLY AUTOMATED FUTURE POWER NETWORKS}

\author{
Minjiang CHEN \\ University of \\ Strathclyde - UK \\ minjiang.chen@ \\ strath.ac.uk \\ Graeme BURT \\ University of \\ Strathclyde - UK \\ graeme.burt@ \\ strath.ac.uk
}

\author{
Victoria CATTERSON \\ University of \\ Strathclyde - UK \\ v.m.catterson@ \\ strath.ac.uk
}

Mattia MARINELLI

Technical University of

Denmark - Denmark

matm@elektro.dtu.dk

\begin{abstract}
Operating power systems is an extremely challenging task, not least because power systems have become highly interconnected, as well as the range of network issues that can occur. It is therefore a necessity to develop decision support systems and visualisation that can effectively support the human operators for decisionmaking in the complex and dynamic environment of future highly automated power system. This paper aims to investigate the decision support functions associated with frequency deviation events for the proposed Web of Cells concept.
\end{abstract}

\section{INTRODUCTION}

Electric power systems have recently experienced unprecedented changes due to the emergence of renewable energy resources, such as wind and photovoltaics, in addition to growing demand. These changes have caused an increase in the size and operational complexity of modern power systems. It is anticipated that in the future, networks will be pushed further towards stability limits and power flow constraints. As a result, human operators may have difficulties in forming a complete and accurate picture of the large-scale interconnected power system region they are responsible for. Moreover, operators should be presented with the data and information that they need to understand the current state of the system and be able to see projected future behaviours. Therefore, acquiring adequate situational awareness (SA) is particularly critical in assisting operators to make the right decisions and respond effectively during system events. It has been shown that several power system blackouts occurred because of operator errors due to insufficient SA [1-3].

In order to address the problems described above, it is very important to develop an advanced decision support system (DSS) to incorporate pertinent information and knowledge to help human operators make control

\author{
Mazheruddin SYED \\ University of \\ Strathclyde - UK \\ mazheruddin.syed@ \\ strath.ac.uk
}

Alexander M.

PROSTEJOVSKY

Technical University of

Denmark - Denmark

alepros@elektro.dtu.dk

\author{
Stephen MCARTHUR \\ University of \\ Strathclyde - UK \\ s.mcarthur@ \\ strath.ac.uk
}

Kai HEUSSEN

Technical University of

Denmark - Denmark

kh@elektro.dtu.dk

decisions quickly and correctly. The DSS aim is to provide SA and, where operator action is required, prioritised alternative solutions to a problem by utilising decision theory techniques. In addition, the DSS should be able to apply the optimal solution if there is no response from the human operator within a certain time, to ensure that the power system recovers from events. There are a variety of DSS applications in the power domain that includes demand response [4], fault diagnosis of generated alarms [5], power system restoration [6], and nuclear power plant operation [7]. Visualisation is critical to relay key information to the operator to better comprehend a situation and to make accurate decisions and faster actions.

This paper considers one of the future network visions called the Web of Cells (WoC), proposed by the ELECTRA (European Liaison on Electricity Committed Towards long-term Research Activity) Integrated Research Programme [8,9]. This vision aims to achieve distributed control of autonomous regions within the power system, called cells. Hence, the future power system is foreseen to drive a move from centralised to a more distributed power system. Developing a decentralized control strategy for a large-scale interconnected power system may be preferable since there is no requirement for global knowledge of the whole power system. Thus, decentralized control may offer efficient, reliable and robust solutions.

The aim of this paper is to explore the functions of the DSS associated with decentralized control during frequency deviation events under the WoC architecture. It demonstrates the required local parameters and measurements that can assist the human operator to resolve incidents by actions taken within the cell, or by requesting support from neighbouring cells.

In this paper, the human operator is intended to mean the power engineer with responsibility for online supervision of the network, and it does not necessarily imply the existence of a physical cell control room. This means that the role of the human operator will inevitably change 
from their current responsibilities. The human operator is responsible for one or more cells, potentially remotely, and the DSS assists operators to ensure emergency situations can be recovered when the system reaches its limits of operation.

\section{FUTURE DISTRIBUTED POWER SYSTEM ARCHITECURE}

The ELECTRA WoC architecture is one particular view of future power systems. A cell is defined as "a group of interconnected loads, distributed energy resources and storage units within well-defined grid boundaries corresponding to a physical portion of the grid and to a confined geographical area" [8]. Under this vision, a larger network can be divided into several cells. Each cell is equipped with a basic set of control functions, and is a peer of other cells and there is no hierarchical control over cells by a superior entity (i.e. system operator). Peer cells will collaborate with each other to allocate resources, such as power exchange via tie lines between cells. Furthermore, a cell is not limited to a particular voltage level, and some cells may span voltage levels. With this in mind, there may not be transmission system operators and distribution system operators, but simply cell operators [9]. Hence, the task of the cell operator is to supervise one or more cells and their automatic control systems to manage network deviations, such as voltage excursions, thermal constraints, and frequency disturbances.

\section{WEB OF CELL FREQUENCY MANAGEMENT}

This paper aims to investigate frequency events within the WoC architecture. Here, frequency is considered a local quantity that is treated independently in order to limit control responses from other cells. Automated data exchange with the rest of the system ensure that global balancing requirements are met. There are three automated services to handle the frequency if it deviates beyond the operational limits: frequency containment control (FCC), balance restoration control (BRC), and balance steering control (BSC) [10]. The descriptions of each control are explained in the following.

\section{Frequency Containment Control}

Once a frequency deviation occurs, FCC aims to stop the rise or fall of frequency and to contain the frequency to a pre-defined value.

\section{Balance Restoration Control}

After the frequency deviation is contained, BRC aims to restore the frequency within set bounds by activating local devices and/or exchanging power with other cells.

\section{Balance Steering Control}

Even although the frequency has been restored within the operational limits by BRC, the reserves used at the BRC stage are fast acting and may be expensive. BSC aims to replace the $\mathrm{BRC}$ reserves with the most economical resources

Two phases of frequency management are considered as shown in Figure 1. One is the procurement phase, where reserves for FCC and BRC services are procured by an algorithm distributed among the cells within the network. The implementation of this algorithm is out of scope of this paper, which focuses instead on the functions of the DSS within the cell. The other is the real-time operation phase which executes these three services within the cell to solve any frequency deviations.

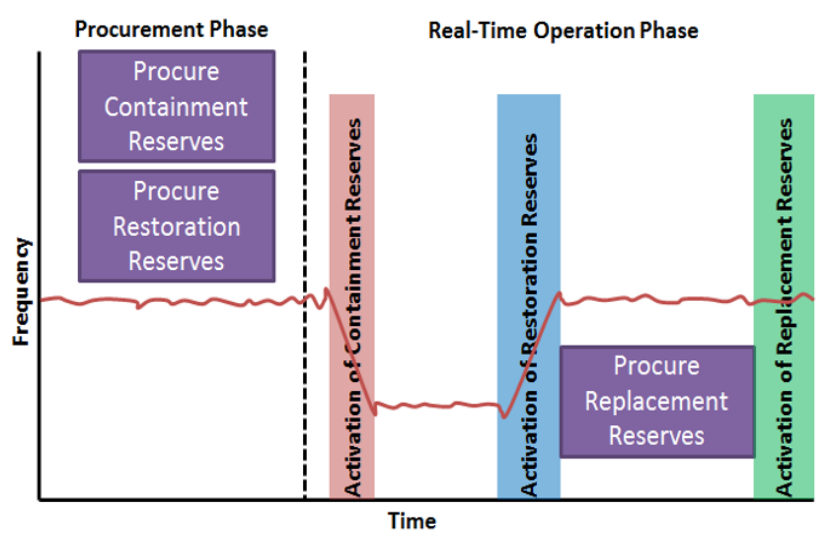

Figure 1. Frequency management in the web of cell

\section{REQUIREMENTS FOR NOVEL DECISION SUPPORT DURING FREQUENCY DEVIATIONS}

Based on the automated WoC frequency management described above, the DSS to support operators has specific requirements that differ from current network management. The human operator should not be required to constantly monitor what the system is doing, but instead should be alerted to changes in status that may eventually lead to problems. However, there should always be the option for the operator to see what the automation is doing. Information regarding the state of the automation should be clearly presented to the human operators.

For frequency management, once a frequency deviation occurs, FCC takes control and reports to the DSS that frequency containment is operational. If FCC is executed automatically as expected, there is no operator action required. After frequency is contained, BRC starts to restore the frequency and reports to the DSS a forecast of when the frequency should return to operational limits. Assuming BRC operates correctly, there is again no operator action required.

If and when these automated control systems fail to take sufficient action, the human operator must be able to resolve the issue. Therefore, the forecast of contained/restored frequency must be compared with online measurements, in order to highlight any failure to 
operate. In such a situation the decision support should guide the operator as to what actions are available, and their expected outcomes. These actions can be automatically prioritised, with the best solution applied automatically after a given time window. In this way full automation is possible, as there are no actions that are really necessary from an operator's point of view. However, operators still require tools for re-prioritising the options, particularly in unusual circumstances such as extreme network events where the operator may have pertinent local information. As a result, the DSS can aid the operator to process situations and provide the list of options for the operator to select.

\section{DECISION SUPPORT DESIGN}

In order to design the decision support for frequency events, there are two frequency scenarios developed here. One is the single frequency event within one cell that is illustrated in Figure 2, and the other is two frequency events in close succession within one cell which is depicted in Figure 3. Each frequency scenario has two decision points where human intervention may improve the outcomes. For each decision point, metrics for selecting between alternative actions to resolve the decision are determined. It is assumed for each scenario that the cell has already procured containment reserves and restoration reserves for FCC and BRC during the procurement phase. This paper only focuses on the realtime operation phase for each scenario.

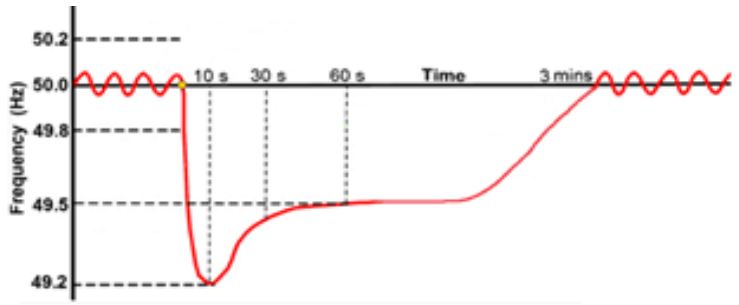

Figure 2. Single frequency event

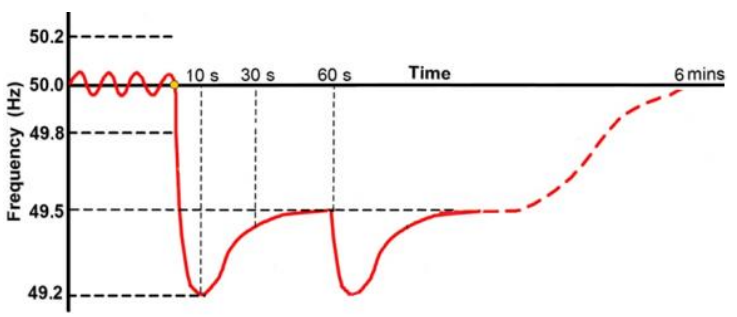

Figure 3. Two frequency events in close succession

\section{Single Frequency Event}

For the single frequency event, it is assumed that the frequency deviation can be mitigated by BRC reserves previously procured during the procurement phase. Hence, the WoC frequency management will automatically return the frequency within operational limits. The intra- and inter-cell messaging required to gather situational awareness data of this event was discussed in [10], where it was highlighted that the DSS of the problem cell will require more detailed information than the DSS of the neighbouring cell. Further to this, the scenario has two key decision points where only the problem cell DSS need be involved.

\section{Procurement of New BRC Reserves}

After frequency is restored, the cell may need to replenish the BRC reserves in case of a future event. The WoC concept includes a periodic planning phase, where neighbouring cells can negotiate to provide each other with support in case of events during real time operation. If this frequency scenario occurs very quickly after the previous planning phase, the cell operator may wish to procure new reserves to mitigate the further loss of generation.

In this scenario, the DSS aims to decide which reserves should be procured for BRC. There are three different metrics that need to be considered. First is the time remaining until the next planning phase. If there is a very short period before the next scheduled planning phase, the operator may prefer to take the risk of operating with depleted reserves, instead of procuring further reserves which could be expensive. The second metric is the available reserves within its own cell and neighbouring cells, with properties of speed of response, cost, and capacity. Some reserves are fast acting (e.g. Hydro) and some reserves are slower and possibly cheaper, but a fast response is needed to restore the frequency during an event. The last metric is that all reserve provision must stay within the tie-line operation margin.

The DSS must trade off these metrics in order to present a prioritised list of options for BRC procurement. It may be the case that the human operator has specific local knowledge that leads them to re-prioritise the options, perhaps to preferentially procure only local reserves, or to avoid typically fast acting reserves that are temporarily responding more slowly. Therefore, the DSS offers suggestions rather than imposing a solution. The operator may choose to delegate the choice to the DSS, or to adjust the list before procurement. A successful outcome would be that the operator is satisfied that sufficient reserves have been procured if needed for BRC for the remainder of this real time operation period.

\section{BSC Replacement of BRC Deployed Reserves}

As the reserves used at the BRC stage are fast acting and therefore expensive, $\mathrm{BSC}$ is responsible for replacing these reserves with the most economical choices. However, the most economical solution is not necessarily the best solution. In this case, the DSS aims to replace the deployed BRC reserves with economical resources. Thus, the metrics applied are available reserves from its own cell and neighbouring cells (speed of respond, cost and capacity) and tie-line operation margins. A successful outcome would be any expensive BRC deployed reserves being replaced with cheaper options, and thereby making them available for any future imbalance event. 


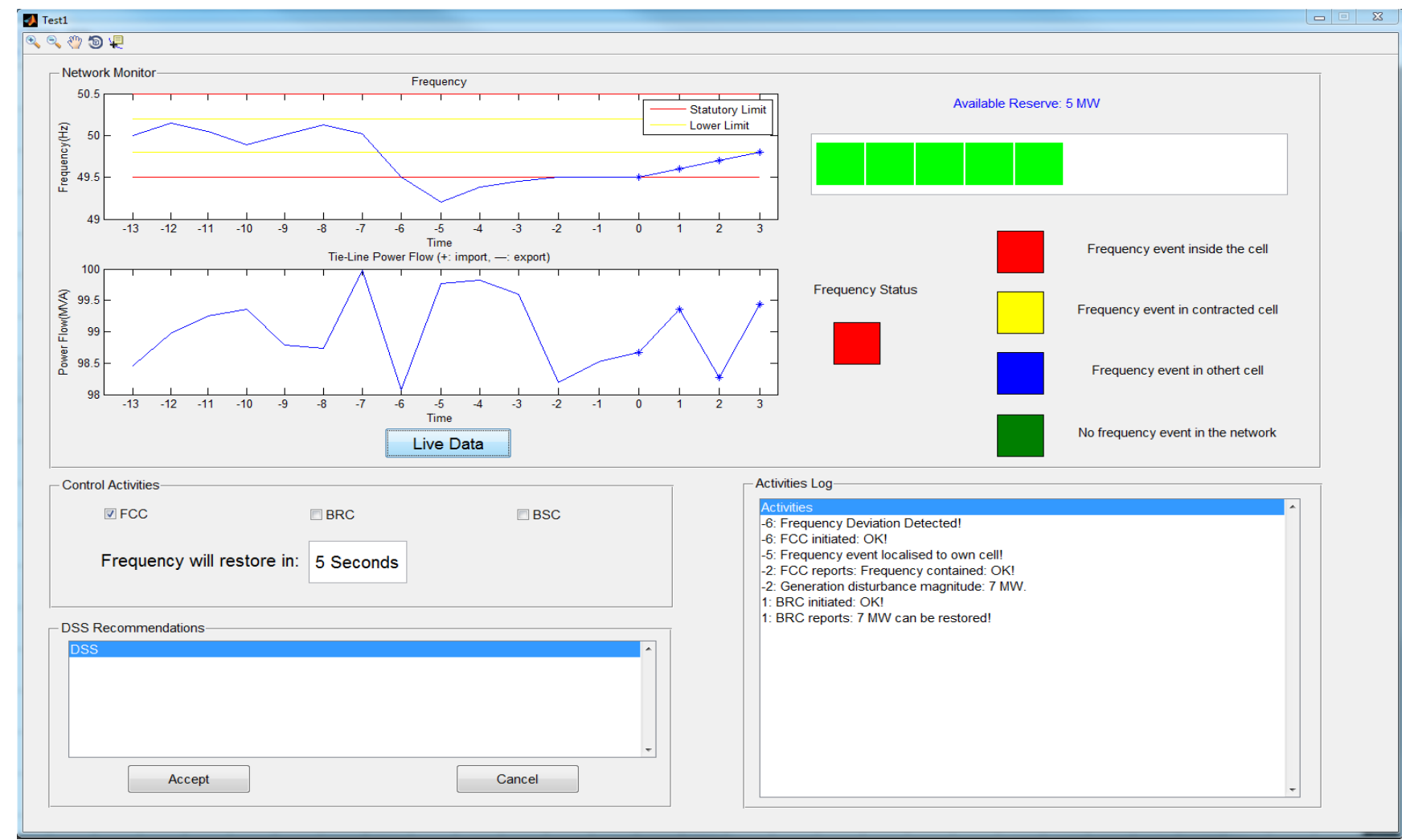

Figure 4. Cell visualization example

\section{Two Frequency Events in Close Succession}

The second scenario involves two frequency events occurring in close time proximity within one cell that disturb the system balance. It is assumed that the combined effect of the events is more significant than the BRC reserves can mitigate. As a result, further action must be taken to procure emergency support and return the frequency within operational limits. As before, the intra- and inter-cell messaging which gathers situational awareness data of this event was discussed in [10].

Response to a Frequency Event Larger than the BRC Reserves Can Handle

The first decision point involves the problem cell operator. After the second frequency deviation is contained, BRC reserves are deployed. However, the frequency does not return within operational limits as the planned reserves do not have the required capacity. At this point, the problem cell contacts its local devices and neighbouring cells to ask how much support can be offered to mitigate this emergency situation.

The DSS aims to restore the frequency within operational limits as soon as possible by considering the available reserves from its own cell and neighbouring cells (speed of respond, cost and capacity) and tie-line operation limits. These options are presented to the operator as in the previous scenario, with a time-out ensuring that the highest priority solution is implemented even in the absence of human input. The successful outcome is balance being restored to the system.

\begin{abstract}
Response to an Emergency Request from a Neighbouring Cell for BRC Support

This decision point is complementary to the previous one, and considers the scenario for the neighbouring cell. The operator of the cell neighbouring the problem cell needs to decide how much support can be committed to the problem cell. There are four different metrics that need to be taken into account. One is the uncommitted reserves. If this cell has spare capacity which is uncommitted, it should generally be offered to the problem cell. However, if this cell has committed reserves, which is the second metric, it may decide to offer these reserves to support the other cell, depending on how soon the next planning phase begins (the third metric). If this real time operation phase finishes in 10 minutes, compared to it finishing in two hours, it is very low risk to offer committed reserves. The last metric is the tie-line operation limit.

As before, the DSS should trade off these metrics to produce alternative plans of how much support from which devices to the problem cell. The prioritised list of options is presented to the operator, who may delegate the choice to the DSS, or select an alternative to the top ranked option. A successful outcome would be where sufficient reserves are offered to the problem cell without compromising this cell's commitments.
\end{abstract}

\section{CELL VISUALIZATION EXAMPLE}

The DSS aims to present a visualization that allows the operator to observe the system state at a glance and 
enable intuitive situational awareness. Some elements of the cell visualisation within the WoC architecture are shown in Figure 4. The frequency deviation is highlighted by colour on the right hand side. Graphs on the left show the status of frequency and tie-line power flow of the cell (only one tie-line interconnected). In addition, it is essential to assist the operator with projections of the system state into the future. In order to do that, a forecast of the planned frequency restoration after containment is shown with a starred line in the frequency status graph (the time 0 indicates the time now and negative time and positive time shows measurements and forecast separately). Moreover, it predicts the time when frequency will be restored underneath the graph.

As already mentioned, the human operator needs to be able to see which automatic control actions are operating. Therefore, check boxes display the status of automated control including FCC, BRC, and BSC. If one of the automated controllers fails, the operator can manually take action to resolve this issue.

In the top right, there is an indicator to show the available reserves within the cell. This is to help the operator to make decisions if the cell needs to procure more BRC reserves for the remaining time period or not. In order to let the operator be aware of what is happening in the network, the bottom right shows a network activity log which records network information, such as location and magnitude of frequency disturbance.

In the bottom left a decision support box lists options for the operator to select. If there is no need for operator action, this box remains blank. The DSS also allows control actions to be input by the operator, because the human operator may know of alternative strategies, or may wish to alter the priority of provided options. Hence, the operator has a certain window of time in which to override the decision support in such a case, after which if no response has been received, the DSS automatically applies the most optimal solution.

\section{CONCLUSION}

This paper has explored the functions of DSS within the WoC architecture. Despite increasing automation, human operators remain an integral part of modern power systems. It is therefore vitally important to provide effective and timely DSS during the real-time operation of power systems. This paper explores the role of DSS in suggesting appropriate decisions within different frequency scenarios based on key metrics. Based on the WoC architecture, the operator must be able to gain situational awareness of the system state, be alerted when a decision can be made, and be offered a prioritised list of suggested actions along with their expected outcomes. With a visualisation of this critical information, the DSS can support an effective response of human operators in challenging situations. As the next step, an appropriate tool, such as constraint satisfaction, optimization algorithms and case base reasoning, will be selected to design the above DSS. Then, DSS will be implemented and tested in the lab.

\section{Acknowledgments}

The work in this paper has been in part supported by the European Commission under the FP7 project ELECTRA (grant no: 609687)

\section{REFERENCES}

[1] M. Panteli, D. S. Kirschen, 2015, "Situation awareness in power systems: Theory, challenges and applications", Electric Power Systems Research, Volume 122, pp. 140-151.

[2] UCTE, 2006, Final Report System Disturbance on 4 November 2006, Available: https://www.entsoe.eu.

[3] U.S.-Canada Power System Outage Task Force, 2004, Final Report on the August 14, 2003 Blackout in the United States and Canada: Causes and Recommendations, Available: http://www.nerc.com.

[4] F. Pedro, and Z. Vale, 2014, "Decision Support Concerning Demand Response Programs Design and Use-A Conceptual Framework and Simulation Tool", Applied Mathematics \& Information Sciences, vol.1, p.161.

[5] G. M. Burt, J. R. McDonald, A. G. King, J. Spiller, D. Brooke and R. Samwell, 1994, "A real-time decision support system for the operation of a $132 \mathrm{kV}$ power network," Control, International Conference on, vol.1, pp. 153-158.

[6] A. Stefanov, C. Liu, M. Sforna, M. Eremia and R. Balaurescu, 2015, "Decision support for restoration of interconnected power systems using tie lines", IET Generation, Transmission \& Distribution, vol.11, pp.1006-1018.

[7] S. J. Lee, K. Mo and P. H. Seong, 2007, "Development of an integrated decision support system to aid the cognitive activities of operators in main control rooms of nuclear power plants", In IEEE Symposium on Computational Intelligence in Multicriteria Decision Making, pp. 146-152.

[8] L. Martini, L. Radaelli, H. Brunner, C. Caerts, A. Morch, S. Hanninen and C. Tornelli, 2015, "ELECTRA IRP Approach to Voltage and Frequency Control for Future Power Systems with High DER Penetration", 23rd International Conference on Electricity Distribution (CIRED).

[9] M. H. Syed, G. M. Burt, J. K. Kok and R. D'Hulst, 2015, “Demand Side Participation for Frequency Containment in the Web of Cells Architecture", International Symposium on Smart Electric Distribution Systems and Technologies (EDST).

[10] A. S. Zaher, V. M. Catterson, M. H. Syed, S. D. J. McArthur, G. M. Burt, M. Chen, M. Marinelli and A. Prostejovsky, 2016, "Enhanced Situational Awareness and Decision Support for Operators of Future Distributed Power Network Architectures," in IEEE PES Innovative Smart Grid Technologies (ISGT Europe) Conference and Exhibition, pp. 1-6. 